\title{
UM ESTUDO SOBRE FUNÇÕES VETORIAIS
}

Antonio da Silva Gomes Júnior, Eugenia Brunilda Opazo Uribe

Universidade Federal de Mato Grosso do Sul, Três Lagoas, MS. E-mail: antonio 3lagoas@hotmail.com Agência de fomento: Programa Institucional de Bolsa de Iniciação à Docência (PIBID), Programa de Educação Tutorial (PET)

\section{RESUMO}

O presente artigo é resultado de uma atividade de pesquisa para a conclusão do Curso de Matemática, visando o estudo das funções vetoriais e sua aplicação à demonstração das Leis de Kepler. A metodologia escolhida foi a apresentação de seminários associada à resolução de exercícios. Foram explorados conceitos de limites, derivadas e integrais de funções vetoriais, bem como alguns resultados importantes. Finalmente foram enunciadas e demonstradas as Leis de Kepler. Concluímos que o trabalho permitiu o aprofundamento de conceitos conhecidos de cálculo, bem como a possibilidade de conhecer tópicos que não são apresentados nas disciplinas regulares do curso.

Palavras-chave: Cálculo Diferencial e Integral; funções Vetoriais; Leis de Kepler

\section{A STUDY ON VECTOR FUNCTIONS}

\begin{abstract}
This article is the result of a research activity to complete math Course, aimed at the study of vector functions and its application to demonstrate the Kepler's Laws. The methodology chosen was the presentation of seminars associated with the resolution of exercises. Were explored concepts of limits, derivatives and integrals of vector functions, as well as some important results. Were explored concepts of limits, derivatives and integrals of vector functions, as well as some important results. Finally stated and demonstrated the laws of Kepler. We conclude that the work has enabled the development of known concepts of calculus, as well as the possibility of meeting topics that are not presented in the regular disciplines of the course.

Keywords: Differential and Integral Calculus; Vector functions; Kepler's laws
\end{abstract}




\section{INTRODUÇÃO}

Segundo Anton (2000), as funções são usadas por matemáticos e por cientistas para descrever as relações entre quantidades variáveis e, assim, desempenham um papel central no cálculo e nas aplicações. No estudo do Cálculo iniciamos com as funções reais de variável real e em seguida trabalhamos com funções de várias variáveis, cujo estudo se justifica porque elas modelam diversos problemas matemáticos além de aparecer naturalmente em outras ciências e na natureza. Podemos estudar também as funções a valores vetoriais, isto é, funções cujos valores são vetores e que são úteis para descrever superfícies e curvas espaciais, bem como para descrever o movimento de objetos no espaço (Stewart, 2007).

O objetivo do presente trabalho é apresentar os resultados de um estudo sobre as funções vetoriais e sua aplicação na demonstração das Leis de Kepler.

\section{METODOLOGIA}

O trabalho foi desenvolvido através de um estudo teórico que incluiu apresentação de seminários semanais e resolução de exercícios.

\section{RESULTADOS}

O trabalho apresenta os resultados de pesquisa desenvolvida para a conclusão do Curso de Licenciatura em Matemática com o intuito de aprofundar os conhecimentos adquiridos durante a graduação. Assim, iniciaremos com uma revisão de conceitos teóricos sobre funções vetoriais incluindo alguns exemplos de aplicação e finalizaremos utilizando estes conceitos para derivar as Leis de Kepler, seguindo o roteiro proposto como projeto aplicado por Stewart 2007.

Denominaremos de função vetorial a uma função cujo domínio é um conjunto de números reais e cuja imagem é um conjunto de vetores. Nosso estudo ficará restrito a funções $\boldsymbol{r}$ cujos valores são vetores tridimensionais, o que significa que para todo número real $t$ no domínio de $\boldsymbol{r}$ existe um único vetor denotado por $\boldsymbol{r}(t)$.

Definição 1: Se $f(t), g(t), h(t)$ são os componentes do vetor $\boldsymbol{r}(t)$, então $f, g$ e $h$ são funções de valor real chamadas funções componentes de $\boldsymbol{r}$ e escrevemos

$$
\boldsymbol{r}(t)=\langle f(t), g(t), h(t)\rangle=f(t) \boldsymbol{i}+g(t) \boldsymbol{j}+h(t) \boldsymbol{k}
$$

Podemos definir o limite e a continuidade de uma função vetorial $\boldsymbol{r}$ a partir da análise de suas funções componentes, como feito nas definições 2 e 3.

Definição 2 : Se $\boldsymbol{r}(t)=\langle f(t), g(t), h(t)\rangle$, então

$$
\lim _{t \rightarrow a} \boldsymbol{r}(t)=\left\langle\lim _{t \rightarrow a} f(t), \lim _{t \rightarrow a} g(t), \lim _{t \rightarrow a} h(t)\right\rangle
$$

desde que os limites das funções componentes existam.

Definição 3: Uma função vetorial $\boldsymbol{r}$ é contínua em $a$ se $\lim _{t \rightarrow a} \boldsymbol{r}(t)=\boldsymbol{r}(a)$. Ou seja $\boldsymbol{r}$ é contínua em $a$ se, e somente se, suas funções componentes $f, g$ e $h$ são contínuas em $a$.

Definição 4: Sejam $f, g$ e $h$ funções reais contínuas em um intervalo $I$. Então o conjunto $C$ de todos os pontos $(x, y, z)$ no espaço para os quais $x=f(t), y=g(t)$ e $z=h(t)$ e $t$ varia no intervalo $I$ é chamado de curva espacial.

Definição 5: Definimos a derivada $\boldsymbol{r}^{\prime}$ de uma função vetorial $\boldsymbol{r}$ como

$$
\frac{d r}{d t}=\boldsymbol{r}^{\prime}(t)=\lim _{h \rightarrow 0} \frac{\boldsymbol{r}(t+h)-\boldsymbol{r}(t)}{h}
$$

se o limite existir. 
O vetor $\boldsymbol{r}^{\prime}(t)$ será chamado de vetor tangente à curva definida por $\boldsymbol{r}$ no ponto $P$, desde que exista $\boldsymbol{r}^{\prime}(t)$ e $\boldsymbol{r}^{\prime}(t) \neq 0$. A reta tangente à curva em $P$ é definida como a reta que passa por $P$ e é paralela ao vetor $\boldsymbol{r}^{\prime}(t)$. Além disso, consideraremos o versor tangente $\boldsymbol{T}(t)=\frac{\boldsymbol{r}^{\prime}(t)}{\left|\boldsymbol{r}^{\prime}(t)\right|^{\circ}}$.

De maneira análoga ao trabalho feito com limite e continuidade, podemos estabelecer um método de obter a derivada de uma função vetorial a partir das derivadas de cada uma das suas componentes, de acordo com o Teorema 1.

Teorema 1: Se $\boldsymbol{r}(t)=\langle f(t), g(t), h(t)\rangle=f(t) \boldsymbol{i}+g(t) \boldsymbol{j}+h(t) \boldsymbol{k}$ onde $f, g$ e $h$ são funções diferenciáveis, então

$$
\boldsymbol{r}^{\prime}(t)=\left\langle f^{\prime}(t), g^{\prime}(t), h^{\prime}(t)\right\rangle=f^{\prime}(t) \boldsymbol{i}+g^{\prime}(t) \boldsymbol{j}+h^{\prime}(t) \boldsymbol{k}
$$

A derivada segunda de uma função vetorial $\boldsymbol{r}$ pode ser obtida de maneira análoga ao caso de funções reais pela derivação da primeira derivada, isto é, $\boldsymbol{r}^{\prime \prime}=\left(\boldsymbol{r}^{\prime}\right)^{\prime}$.

Definição 6: Uma curva dada por uma função vetorial $\boldsymbol{r}(t)$ em um intervalo $I$ é denominada lisa se $\boldsymbol{r}^{\prime}$ for contínua e $\boldsymbol{r}^{\prime}(t) \neq 0$ (exceto possivelmente nos limites do intervalo $I$ ).

Observemos que, se a curva que não é lisa e é formada por um número finito de pedaços lisos, ela será denominada lisa por partes.

No próximo teorema estabelecemos as regras de diferenciação das funções vetoriais incluindo a regra da cadeia, observemos também que cada uma das regras de diferenciação para funções reais têm uma regra equivalente para o caso das funções vetoriais.

Teorema 2: Suponhamos que $\boldsymbol{u}$ e $\boldsymbol{v}$ sejam funções vetoriais diferenciáveis, $c$ seja um escalar e $f$, uma função real. Pode ser demonstrado que,

$$
\begin{aligned}
& \text { i) } \frac{d}{d t}[\boldsymbol{u}(t)+\boldsymbol{v}(t)]=\boldsymbol{u}^{\prime}(t)+\boldsymbol{v}^{\prime}(t), \\
& \text { ii) } \frac{d}{d t}[c \boldsymbol{u}(t)]=c \boldsymbol{u}^{\prime}(t) \\
& \text { iii) } \frac{d}{d t}[f(t) \boldsymbol{u}(t)]=f^{\prime}(t) \boldsymbol{u}(t)+f(t) \boldsymbol{u}^{\prime}(t) \\
& \text { iv) } \frac{d}{d t}[\boldsymbol{u}(t) \cdot \boldsymbol{v}(t)]=\boldsymbol{u}^{\prime}(t) \cdot \boldsymbol{v}(t)+\boldsymbol{u}(t) \cdot \boldsymbol{v}^{\prime}(t) \\
& \text { v) } \frac{d}{d t}[\boldsymbol{u}(t) \times \boldsymbol{v}(t)]=\boldsymbol{u}^{\prime}(t) \times \boldsymbol{v}(t)+\boldsymbol{u}(t) \times \boldsymbol{v}^{\prime}(t) \\
& \text { vi) } \frac{d}{d t}[\boldsymbol{u}(f(t))]=f^{\prime}(t) \boldsymbol{u}^{\prime}(f(t))
\end{aligned}
$$

Teorema 3: Se $|\boldsymbol{r}(t)|=c$, onde $c$ é uma constante, então $\boldsymbol{r}^{\prime}(t)$ é ortogonal a $\boldsymbol{r}(t)$ para todo $t$. Demonstração: Como $\boldsymbol{r}(t) \cdot \boldsymbol{r}(t)=|\boldsymbol{r}(t)|^{2}=c^{2}$ e $c^{2}$ é uma constante, então do item (iv) do Teorema 2, temos

$$
\frac{d}{d t}[\boldsymbol{r}(t) \cdot \boldsymbol{r}(t)]=\boldsymbol{r}^{\prime}(t) \cdot \boldsymbol{r}(t)+\boldsymbol{r}(t) \cdot \boldsymbol{r}^{\prime}(t)=2 \boldsymbol{r}^{\prime}(t) \cdot \boldsymbol{r}(t)=0
$$

Então, $\boldsymbol{r}^{\prime}(t) \cdot \boldsymbol{r}(t)=0$, o que implica que $\boldsymbol{r}^{\prime}(t)$ é ortogonal a $\boldsymbol{r}(t)$.

Analogamente ao trabalho feito com derivadas, podemos definir a integral de funções vetoriais através da integral das suas funções componentes.

Definição 7: Seja $\boldsymbol{r}(t)=\langle f(t), g(t), h(t)\rangle=f(t) \boldsymbol{i}+g(t) \boldsymbol{j}+h(t) \boldsymbol{k}$ onde $f, g$ e $h$ são funções contínuas em $[a, b]$. Definimos a integral definida no intervalo $[a, b]$ como a integral de suas funções componentes $f, g$ e $h$ como se segue

$$
\int_{a}^{b} \boldsymbol{r}(t) d t=\left\langle\int_{a}^{b} f(t) d t, \int_{a}^{b} g(t) d t, \int_{a}^{b} h(t) d t\right\rangle
$$

Ou seja, para $\boldsymbol{r}(t)$ ser integrável todas as funções componentes $f, g$ e $h$ devem ser integráveis. 
Teorema 3: Seja $\boldsymbol{r}(t)=\langle f(t), g(t), h(t)\rangle, a \leq t \leq b$, onde $f^{\prime}, g^{\prime}$ e $h^{\prime}$ são funções contínuas. Se a curva não se intercepta quando o parâmetro $t$ cresce pode ser mostrado que o comprimento $L$ de uma curva $\boldsymbol{r}(t)$ é

$$
L=\int_{a}^{b} \sqrt{\left[f^{\prime}(t)\right]^{2}+\left[g^{\prime}(t)\right]^{2}+\left[h^{\prime}(t)\right]^{2}} d t
$$

A curvatura de $C$ em um ponto é a medida de quão rápido a curva muda de direção no ponto.

Definição 8: A curvatura de uma curva é $k=\left|\frac{d T}{d s}\right|$ onde $\boldsymbol{T}$ é o versor da tangente.

Teorema 4: A curvatura de uma curva dada pela função vetorial $r$ é

$$
k(t)=\frac{\left|\boldsymbol{r}^{\prime}(t) \times \boldsymbol{r}^{\prime \prime}(t)\right|}{\left|\boldsymbol{r}^{\prime}(t)\right|^{3}}
$$

Em um ponto dado de uma curva lisa $\boldsymbol{r}(t)$ existem muitos vetores que são ortogonais ao versor da tangente $\boldsymbol{T}(t)$, por exemplo, $\boldsymbol{T}^{\prime}(t)$. No entanto, $\boldsymbol{T}^{\prime}(t)$ pode não ser unitário.

Definição 9: Se $\boldsymbol{r}^{\prime}$ for lisa, definiremos o vetor normal unitário como

$$
\boldsymbol{N}(t)=\frac{\boldsymbol{T}^{\prime}(t)}{\left|\boldsymbol{T}^{\prime}(t)\right|}
$$

E o vetor $\boldsymbol{B}(t)=\boldsymbol{T}(t) \times \boldsymbol{N}(t)$, perpendicular a $\boldsymbol{T}$ e $\boldsymbol{N}$ e unitário como vetor binormal.

Podemos aplicar os conceitos de vetores tangentes e normal e curvatura para estudar o movimento de objetos, sua velocidade e sua aceleração, quando esses se movem ao longo de uma curva espacial.

Definição 10: Se $\boldsymbol{r}(t)$ for o vetor posição de uma partícula ao longo de uma curva, então a velocidade, a aceleração e a rapidez da partícula no instante $t$ são definidas por

$$
\begin{gathered}
\text { Velocidade } \boldsymbol{v}(t)=\frac{d r}{d t}=\boldsymbol{r}^{\prime}(t) \\
\text { Aceleração } \boldsymbol{a}(t)=\frac{d v}{d t}=\boldsymbol{r}^{\prime \prime}(t) \\
\text { Rapidez }|\boldsymbol{v}(t)|=\frac{d s}{d t}
\end{gathered}
$$

Quando estudamos o movimento de uma partícula, é frequentemente útil decompor a aceleração em duas componentes, uma na direção da tangente e outra na direção da normal.

\section{Primeira Lei de Kepler: Um planeta gira em torno do Sol em uma Orbita elíptica com o Sol em um dos focos.}

Segundo Stewart (2007), o fato da força gravitacional do Sol sobre um planeta ser muito maior que as forças exercidas por outros astros, podemos ignorar todos os outros corpos do universo, exceto o Sol e um planeta girando em torno dele.

Consideraremos um sistema de coordenadas com origem no Sol e $\mathbf{r}=\boldsymbol{r}(t)$ o vetor posição do planeta. Assim, o vetor velocidade será $\boldsymbol{v}=\mathbf{r}^{\prime}$ e o vetor aceleração será $\boldsymbol{a}=\mathbf{r}^{\prime \prime}$.

Para podermos fazer as demonstrações precisaremos utilizar duas leis físicas devidas a Isaac Newton:

$$
\begin{gathered}
\boldsymbol{F}=m \boldsymbol{a} \text { (Segunda Lei do Movimento) } \\
\boldsymbol{F}=-\frac{G M m}{r^{3}} \mathbf{r}=-\frac{G M m}{r^{2}} \boldsymbol{u} \text { (Lei de Gravitação Universal), }
\end{gathered}
$$

onde $\boldsymbol{F}$ é a força da gravidade sobre o planeta, $m$ e $M$ são as massas do planeta e do Sol, $G$ é a constante gravitacional, $r=|\mathbf{r}|$ e $\boldsymbol{u}=(1 / r) \mathbf{r}$ é o vetor $\mathbf{r}$.

Primeiro mostraremos que o planeta se move em um plano. Igualando as duas expressões para $\boldsymbol{F}$ obtemos que $\boldsymbol{a}=-\frac{G M}{\boldsymbol{r}^{3}} \mathbf{r}, \mathrm{e}$, portanto $\boldsymbol{a}$ é paralelo a $\boldsymbol{r}$. Teremos então que $\boldsymbol{r} \times \boldsymbol{a}=0$. Do Teorema 2 item (v) podemos escrever 


$$
\frac{d}{d t}(\mathbf{r} \times \boldsymbol{v})=\mathbf{r}^{\prime} \times \boldsymbol{v}+\mathbf{r} \times \boldsymbol{v}^{\prime}=\boldsymbol{v} \times \boldsymbol{v}+\mathbf{r} \times \boldsymbol{a}=0+0=0
$$

Assim, $\mathbf{r} \times \boldsymbol{v}=\mathbf{h}$, onde $\mathbf{h}$ é um vetor constante. ( $\mathbf{h} \neq 0$, ou seja, $\boldsymbol{r}$ e $\boldsymbol{v}$ não são paralelos). Isso significa que o vetor $\boldsymbol{r}=\boldsymbol{r}(t)$ é perpendicular a $\mathbf{h}$ para todos os valores de $t$, portanto o planeta está sempre em um plano que passa pela origem é perpendicular a $\mathbf{h}$. Dessa forma, a órbita do planeta é uma curva plana.

Para provar a Primeira Lei de Kepler, vamos reescrever o vetor $\mathbf{h}$ da seguinte forma:

$$
\begin{aligned}
\mathbf{h} & =\mathbf{r} \times \boldsymbol{v}=\mathbf{r} \times \mathbf{r}^{\prime}=r \boldsymbol{u} \times(r \boldsymbol{u})^{\prime}=r \boldsymbol{u} \times\left(r \boldsymbol{u}^{\prime}+r^{\prime} \boldsymbol{u}\right) \\
& =r^{2}\left(\boldsymbol{u} \times \boldsymbol{u}^{\prime}\right)+r r^{\prime}(\boldsymbol{u} \times \boldsymbol{u})=r^{2}\left(\boldsymbol{u} \times \boldsymbol{u}^{\prime}\right)
\end{aligned}
$$

Então, $\boldsymbol{a} \times \mathbf{h}=-\frac{G M}{r^{2}} \boldsymbol{u} \times\left(r^{2} \boldsymbol{u} \times \boldsymbol{u}^{\prime}\right)=-G M \boldsymbol{u} \times\left(\boldsymbol{u} \times \boldsymbol{u}^{\prime}\right)=-G M\left[\left(\boldsymbol{u} \cdot \boldsymbol{u}^{\prime}\right) \boldsymbol{u}-(\boldsymbol{u} \cdot \boldsymbol{u}) \boldsymbol{u}^{\prime}\right]$ Mas $\boldsymbol{u} \cdot \boldsymbol{u}=|\boldsymbol{u}|^{2}=1$ e, como $|\boldsymbol{u}(t)|=1$ segue do Teorema 3 que $\boldsymbol{u} \cdot \boldsymbol{u}^{\prime}=0$. Portanto, $\boldsymbol{a} \times \mathbf{h}=G M \boldsymbol{u}^{\prime}$.

Então, $(\boldsymbol{v} \times \mathbf{h})^{\prime}=\boldsymbol{v}^{\prime} \times \mathbf{h}+\boldsymbol{v} \times \mathbf{h}^{\prime}=\boldsymbol{a} \times \mathbf{h}+0=G M \boldsymbol{u}^{\prime}$. Integrando ambos os lados da igualdade obtêm $\boldsymbol{v} \times \boldsymbol{h}=G M \boldsymbol{u}+\boldsymbol{c}\left({ }^{*}\right)$ onde $\boldsymbol{c}$ é um vetor constante.

Por conveniência escolheremos os eixos coordenados de forma que o vetor da base padrão $\boldsymbol{k}$ aponte na direção do vetor $\boldsymbol{h}$. O planeta se move assim no plano $x y$. Como $\boldsymbol{v} \times \boldsymbol{h}$ e $\boldsymbol{u}$ são perpendiculares a $\boldsymbol{h}$, a equação $\left({ }^{*}\right)$ mostra que $\boldsymbol{c}$ pertence ao plano $x y$. Isso significa que podemos escolher os eixos $x$ e $y$ de forma que $\boldsymbol{i}$ esteja na direção de $\boldsymbol{c}$.

Agora, se $\theta$ é o ângulo entre $\boldsymbol{c}$ e $\mathbf{r}$, então $(r, \theta)$ são as coordenadas polares do planeta. Fazendo produto escalar de $\boldsymbol{r}$ pela equação $(*)$, teremos

$$
\mathbf{r} \cdot(\boldsymbol{v} \times \mathbf{h})=\boldsymbol{r} \cdot(G M \boldsymbol{u}+\boldsymbol{c})=G M \boldsymbol{r} \cdot \boldsymbol{u}+\boldsymbol{r} \cdot \boldsymbol{c} .
$$

Mas, $\boldsymbol{r} \cdot \boldsymbol{c}=|\mathbf{r}||\boldsymbol{c}| \cos \theta$ assim $\mathbf{r} \cdot(\boldsymbol{v} \times \mathbf{h})=G M r \boldsymbol{u} \cdot \boldsymbol{u}+|\mathbf{r}||\boldsymbol{c}| \cos \theta=G M r+r c \cos \theta$ onde $c=|c|$. Então,

$$
r=\frac{\mathbf{r} \cdot(\boldsymbol{v} \times \mathbf{h})}{G M+c \cos \theta}=\frac{1}{G M} \frac{\mathbf{r} \cdot(\boldsymbol{v} \times \mathbf{h})}{(1+e \cos \theta)}
$$

onde $e=c / G M$. Mas, $r \cdot(\boldsymbol{v} \times \mathbf{h})=(\boldsymbol{r} \times \boldsymbol{v}) \cdot \mathbf{h}=\mathbf{h} \cdot \mathbf{h}=|\mathbf{h}|^{2}=h^{2}$ onde $h=|\mathbf{h}|$. Desse modo,

Escrevendo $d=h^{2} / c$, obtemos a equação

$$
r=\frac{h^{2} / G M}{1+e \cos \theta}=\frac{e h^{2} / c}{1+e \cos \theta}
$$

$$
r=\frac{e d}{1+e \cos \theta}
$$

que representa a forma polar da equação da seção cônica com foco na origem e excentricidade $e$. Como sabemos que a órbita de um planeta é uma curva fechada podemos concluir que a órbita precisa ser uma elipse.

\section{Segunda Lei de Kepler: A reta que liga o sol a um planeta percorre áreas iguais em intervalos de tempos iguais.}

Em coordenadas polares podemos escrever que $\boldsymbol{r}=r \cos \theta \boldsymbol{i}+r \operatorname{sen} \theta \boldsymbol{j}$.

Ainda, $\boldsymbol{h}=\alpha \boldsymbol{k}$ onde $\alpha>0$. Assim, substituindo em $\boldsymbol{h}$ obtemos,

$$
\begin{aligned}
\boldsymbol{h}=\boldsymbol{r} \times \boldsymbol{v} & =\boldsymbol{r} \times \boldsymbol{r}^{\prime} \\
& =(r \cos \theta \boldsymbol{i}+r \operatorname{sen} \theta \boldsymbol{j}) \times\left[\left(r^{\prime} \cos \theta-r \operatorname{sen} \theta \frac{d \theta}{d t}\right) \boldsymbol{i}+\left(r^{\prime} \operatorname{sen} \theta+r \cos \theta \frac{d \theta}{d t}\right) \boldsymbol{j}\right] \\
& =\left[r r^{\prime} \operatorname{sen} \theta \cos \theta+r^{2} \cos ^{2} \theta \frac{d \theta}{d t}-\left(r r^{\prime} \operatorname{sen} \theta \cos \theta-r^{2} \operatorname{sen}^{2} \theta \frac{d \theta}{d t}\right) \boldsymbol{k}\right] \\
& =r^{2} \frac{d \theta}{d t} \boldsymbol{k} \quad\left({ }^{* *}\right)
\end{aligned}
$$

Como $\boldsymbol{h}=\alpha \boldsymbol{k}, \alpha>0$ então $\alpha=|\boldsymbol{h}|$. Comparando com equação $\left({ }^{* *}\right)$ temos que $\alpha=|\boldsymbol{h}|=r^{2} \frac{d \theta}{d t}$ Assim, $h=r^{2} \frac{d \theta}{d t}$, onde $h=|\boldsymbol{h}|$. 
Consideremos agora $A=A(t)$ como sendo a área percorrida pelo vetor radial $r=r(t)$ no intervalo de tempo $\left[t_{0}, t\right]$. A área $A$ é dada por $A(t)=\frac{1}{2} \int_{t_{0}}^{t} r^{2} d \theta$, assim, derivando ambos os lados desta equação, obtemos

$$
\frac{d A}{d \theta}=\frac{1}{2} r^{2}
$$

Mas, utilizando a regra da cadeia e substituindo na equação acima, teremos

Logo, substituindo $h$ na equação acima obtemos

$$
\frac{d A}{d t}=\frac{d A}{d \theta} \frac{d \theta}{d t}=\frac{1}{2} r^{2} \frac{d \theta}{d t}
$$

$$
\frac{d A}{d t}=\frac{1}{2} h
$$

Essa equação nos diz que razão pelo qual $A$ é percorrida é constante e prova a 2a Lei de Kepler.

III. Terceira Lei de Kepler: $O$ quadrado do período de revolução de um planeta é proporcional ao cubo do comprimento do maior eixo de sua órbita.

Seja $T$ o período de um planeta em torno do Sol, ou seja, $T$ é o tempo requerido para o planeta dar uma volta completa em torno do Sol, através de uma órbita elíptica. Suponhamos também que os comprimentos dos eixos maior e menor da elipse sejam $2 a$ e $2 b$.

Da segunda Lei de Kepler, obtemos $\frac{d A}{d t}=\frac{1}{2} h$, e assim, integrando ambos os membros da igualdade obtemos $A(t)=\frac{1}{2} h t+c$. Mas $A(0)=0$ então, $0=A(0)=\frac{1}{2} h 0+c=0+c=c$. Logo, $A(t)=\frac{1}{2} h t$.

Como $T$ é o tempo para o planeta dar uma volta completa em torno do sol, concluímos que a área no tempo $T$ é a área da elipse com comprimentos de eixos definidos acima. Assim, $A(T)=\pi a b$. Da equação (\#) e da área da elipse $A(T)$ obtemos,

$$
A(T)=\frac{1}{2} h T=\pi a b \quad \Leftrightarrow \quad T=\frac{2 \pi a b}{h}
$$

Na primeira lei de Kepler chamamos de $e=c / G M$ e $d=h^{2} / c$. Dessas igualdades obtemos que $e d=h^{2} / G M$. Mas, sabemos $a=\left(e d / 1-e^{2}\right)$ e $\left(1-e^{2}\right)=b^{2} / a^{2}$. Substituindo a primeira igualdade na segunda obtemos que $e d=a\left(1-e^{2}\right)=a \frac{b^{2}}{a^{2}}=\frac{b^{2}}{a}$. No entanto, $e d=h^{2} / G M=\frac{b^{2}}{a}$. Elevando ao quadrado a equação (\#\#) e substituindo $h^{2}=b^{2} G M / a$ obtemos finalmente que

$$
T^{2}=\frac{4 \pi^{2} b^{2}}{h^{2}} a^{2} \Leftrightarrow T^{2}=\frac{4 \pi^{2} b^{2}}{\frac{b^{2} G M}{a}} a^{2} \Leftrightarrow T^{2}=\frac{4 \pi^{2} b^{2}}{b^{2} G M} a^{3} \Leftrightarrow T^{2}=\frac{4 \pi^{2}}{G M} a^{3}
$$

Portanto, o quadrado de $T$ (período de revolução de um planeta) é proporcional ao cubo de $a$ (comprimento do maior eixo da elipse).

\section{DISCUSSÃO}

Através da resolução de exercícios e seminários apresentados durante o desenvolvimento do trabalho foi possível perceber como os conceitos aprendidos nas mais diversas disciplinas de Matemática vão aparecendo e se misturando aos novos conceitos. Para atingir o objetivo do trabalho utilizamos conhecimentos aprendidos em cálculo de uma e várias variáveis, geometria, trigonometria, vetores e geometria analítica, física, entre outras.

\section{CONCLUSÃO}

Consideramos nosso objetivo atingido, no sentido de ter conseguido explorar os conceitos relacionados a funções vetoriais e associar estes conceitos ao estudo das Leis de Kepler. Assim, o trabalho realizado ajudou a aprofundar os conhecimentos e ao mesmo tempo perceber o cálculo como uma disciplina integradora na qual são utilizados diversos conceitos matemáticos e inclusive físicos, sendo que alguns deles foram aprendidos em disciplinas mais elementares. 


\section{REFERÊNCIAS}

ANTON, H. Cálculo, um novo horizonte. 6ạ. ed. Porto Alegre: Bookman, 2000.

BIANCHINI, W. Aprendendo Calculo de Várias Variáveis. 2016. Disponível em: <http://www.im.ufrj.br/waldecir>. Consultado em: 16/08/2016.

STEWART, J. Cálculo, volume 2. São Paulo: Thomson Learning, 2007. 